Revue des patrimoines

37 | 2018

Jardins collectifs : de l'abbé Lemire aux jardins d'insertion. Typologies - Expériences - Enjeux de conservation

\title{
Les jardins collectifs ont-ils droit de cité ?
}

\section{Bruno Marmiroli}

\section{(2) OpenEdition}

\section{Journals}

Édition électronique

URL : http://journals.openedition.org/insitu/19352

DOI : 10.4000/insitu. 19352

ISSN : 1630-7305

\section{Éditeur}

Ministère de la culture

\section{Référence électronique}

Bruno Marmiroli, « Les jardins collectifs ont-ils droit de cité ? », In Situ [En ligne], 37 | 2018, mis en ligne le 19 décembre 2018, consulté le 01 mai 2019. URL : http://journals.openedition.org/insitu/19352 ; DOI : 10.4000/insitu.19352

Ce document a été généré automatiquement le 1 mai 2019.

\section{c) () $\Theta$}

In Situ Revues des patrimoines est mis à disposition selon les termes de la licence Creative Commons Attribution - Pas d'Utilisation Commerciale - Pas de Modification 4.0 International. 


\title{
Les jardins collectifs ont-ils droit de cité ?
}

\author{
Bruno Marmiroli
}

\section{Les droits du jardin collectif}

1 Cet article reprend et complète un précédent travail autour du droit au jardin ${ }^{1}$, destiné à rendre compte des difficultés rencontrées pour protéger les jardins « collectifs ", dès lors qu'ils sont implantés dans un territoire soumis à une pression foncière.

2 La notion de jardin "collectif» regroupe différents types de jardins, qu'ils soient familiaux, ouvriers, communautaires, partagés, d'insertion... gérés par un groupe d'habitants organisé sous forme associative ou non. Pour contribuer à la compréhension du rôle et de l'usage du jardin collectif dans l'espace urbain, il faut évoquer l'histoire de l'émergence puis de l'essor des jardins familiaux et de leur mutation récente au sein d'un corpus plus vaste, qui inscrit le jardin et la pratique du jardinage dans la fabrication du territoire métropolitain. Tout le monde s'accorde sur les vertus du jardinage, pratique qui transcenderait les clivages sociaux pour réunir, au sein d'un espace défini, l'ouvrier et le cadre, œuvrant la bêche à la main pour la plus belle tomate ou la meilleure fraise. Chacun reconnaît les bienfaits d'un air sain et pur, d'une nature domestiquée, d'un développement «durable » ou d'une ville « verte ». Ces slogans inscrivent le jardin dans un cadre sympathique et bienveillant mais ils restent éloignés des réalités orientées par les documents de planification urbaine censés incarner des projets politiques. Avant de revenir sur les aménités environnementales du jardin collectif dans l'espace urbain, nous proposons donc de confronter des notions moins édulcorées, en commençant par nous interroger sur la nature des protections dont il bénéficie, tant sa reconnaissance ne lui semble d'aucun secours dès lors que l'extension urbaine menace. La précarité de l'exercice semble bien être une condition préalable aux projets de jardins dans l'espace public, même si certains, regroupés comme pour faire face à l'adversité, semblent mieux armés pour y échapper. Dans un second temps, nous proposerons quelques pistes pour que le jardin collectif soit reconnu en se fondant sur les services écologiques et sociaux 
qu'il rend, sans omettre sa dimension patrimoniale, liée à l'identification du jardin comme lieu d'apprentissage, d'expérimentation et de transmission des savoirs et des plantes.

3 La catégorie des jardins collectifs, initialement connue sous le vocable de «jardins familiaux ", a pour elle une longue histoire ponctuée de personnages célèbres, de préceptes idéologiques et d'effluves de lutte des classes dont il faut rappeler les grandes lignes. Les jardins "ouvriers" naissent avec la révolution industrielle, autour des structures d'habitat collectif portées par le socialisme utopique du début du XIX ${ }^{e}$ siècle. Les phalanstères de Joseph Fourier (1768-1830) figurent parmi les exemples les plus connus de vie communautaire associant les jardins aux autres espaces collectifs. L'idée, qui se développe dans les bassins industriels du nord et de l'est de la France et qui existait déjà dans d'autres pays d'Europe, s'incarne véritablement à travers les figures du père Félix Volpette (1856-1900) ou de l'abbé Jules Lemire (1853-1928), à l'origine de l'invention officielle des « jardins ouvriers » en 1896. Lemire, créateur de la Ligue du coin de terre et du foyer, organe de mise en œuvre de la politique de développement des jardins ouvriers, résumait son œuvre en une phrase : «Étudier, propager et réaliser par les moyens en son pouvoir toutes les mesures propres à établir la famille sur la base naturelle et divine qui est la possession de la terre et du foyer ${ }^{2}$."

Député du Nord du Parti démocrate-chrétien, Jules Lemire se fait le chantre des vertus de l'agriculture et du jardinage, valeurs véritables s'opposant aux vices de la ville et de l'usine. Le glissement vers les valeurs morales n'est plus loin, dès lors que l'absence supposée de lien du sol se confond avec celle de la foi. Ceux qui jardinent, en retrouvant des racines perdues, qu'elles soient religieuses, symboliques ou patriotiques, se tiennent de fait à l'écart des lieux de débauche. Le nombre de jardins ouvriers augmente rapidement, la France en compte 6453 en 1903, 12000 en 1906, et les archives de la Ligue en mentionnent 17825 en $1912^{3}$. Le jardin prôné par l'abbé Lemire est le cadre d'épanouissement d'un "patriotisme, fleurs des âmes à côté des fleurs des parterres " ${ }^{4}$ bien plus qu'un lieu de pratiques potagères.

Dès les premiers congrès se pose la question du rapport au foncier. L'acquisition reste le meilleur moyen de se prémunir des évictions et d'œuvrer à long terme. La Ligue l'a bien compris puisqu'elle acquiert, en 1906, un statut comparable aux sociétés d'habitations bon marché (incitation des communes et départements par des prêts, cessions de terrains à des conditions avantageuses...). D'autres œuvres s'implantent sur des terrains appartenant à des compagnies ou même au Génie militaire, via la rédaction de baux parfois révocables. Une forme de précarité qui permet, une fois encore, à la Ligue de militer pour l'affiliation. Maurice de Vilmorin, président de la Société nationale d'horticulture et membre de la célèbre famille de grainetiers, apparaît également dans le paysage des congrès et ne manque pas de rappeler que seul l'usage des variétés de légumes les plus productives et les plus robustes, l'usage des engrais minéraux... permettent d'obtenir de beaux jardins. Et c'est tout aussi naturellement que peut s'opérer le rapprochement entre les sociétés d'horticulture qui regroupent des passionnés de l'art du jardin, à l'image des sociétés savantes du XIX siècle, et les sociétés de jardins ouvriers, les premières prodiguant conseils et formations au cours d'«amicales causeries ». Non sans une certaine condescendance, le rapprochement, qui s'apparente à une œuvre charitable, permet à la bonne société de rendre visite aux tenanciers ${ }^{6}$ de jardins ouvriers, allant jusqu'à remettre quelques médailles aux plus assidus. Nous retrouverons la visite au jardin dans un tout autre cadre, celui de la campagne "Visitez un jardin », lancée en 
1986, qui intègre les jardins familiaux aux parcours de découverte des jardins publics, participant de leur élévation au rang d'espace vert digne d'être vu. L'idée selon laquelle les parcelles n'étaient pas présentables a longtemps été un motif de suppression de jardins dont l'esthétique était incompatible avec le dessin des nouveaux quartiers et la Ligue a œuvré pour encadrer les pratiques, au moyen de règlements stricts. Le rôle politique des orientations de la Ligue est évoqué dès sa constitution, en écartant l'ouvrier des lieux de réunion syndicale, en reliant systématiquement l'accès au lopin au respect d'un règlement qui dépasse largement le cadre du jardin et ira jusqu'à retirer les parcelles aux jeunes couples restés plus de trois ans sans descendance...

6 Réunies à partir de $1952^{7}$ sous le vocable unique de «jardins familiaux », effaçant l'héritage prolétaire des débuts puis l'idéologie paternaliste associée à l'essor des jardins ouvriers sous le gouvernement de Vichy ${ }^{8}$, les différentes associations se regroupent en Fédération nationale des jardins familiaux, bénéficiant d'exonérations fiscales. De sociétaire, le jardinier devient locataire soumis à une somme de règles qui perdure : les plantes sauvages (sans autre précision quant à leur définition) ne sont pas acceptées ${ }^{9}$, les pratiques de jardinage et les comportements individuels très encadrés (pas de barbecues, pas d'interventions plastiques sur les clôtures imposées par la commune, pas de constructions d'abris ou tonnelles...) allant jusqu'aux choix des variétés ou aux préconisations esthétiques concernant l'aménagement du lieu. Il n'est pas rare de trouver dans les règlements récents la recommandation appuyée d'un fournisseur attitré de semences ou d'engrais et l'on peut parfois s'interroger sur la capacité de ce type de jardins à participer du développement d'un jardinage biologique, de la préservation de variétés anciennes, de la sensibilisation aux espèces locales ou même, et plus simplement, de la liberté du jardinier en son jardin.

7 La loi du 10 novembre 1976, portée par le député Jean Royer ${ }^{10}$, fait entrer les jardins familiaux dans une nouvelle ère qui voit leur protection renforcée : droit de préemption des SAFER ${ }^{11}$ et des collectivités locales, reconnaissance de leur caractère d'équipement social et de loisir, imposition, en cas d'expropriation, d'une réinstallation des jardins... Cette loi, qui fait suite à plusieurs vaines tentatives de Jean Royer de permettre l'assimilation des jardins familiaux à des espaces verts, reste le socle actuel sur lequel repose le cadre législatif destiné à les protéger. Mieux intégrés dans la politique d'aménagement du territoire, pris en compte dans les plans d'urbanisme, bénéficiant d'aides diverses, les groupes de jardins familiaux, gérés par le ministère de l'Environnement et de la Qualité de vie, semblent connaître une nouvelle naissance ${ }^{12}$. La décentralisation mettra un terme aux avancées en renvoyant aux collectivités locales le soin de gérer les espaces et d'octroyer les subventions : « Pour être légitimes, ces mesures n'en pénalisaient pas moins le mouvement des jardins familiaux, tout particulièrement dans les régions où, faiblement représenté ou structuré, il était peu en mesure de peser sur une décision encore plus nécessaire qu'ailleurs ${ }^{13}$.» (fig. 1) 


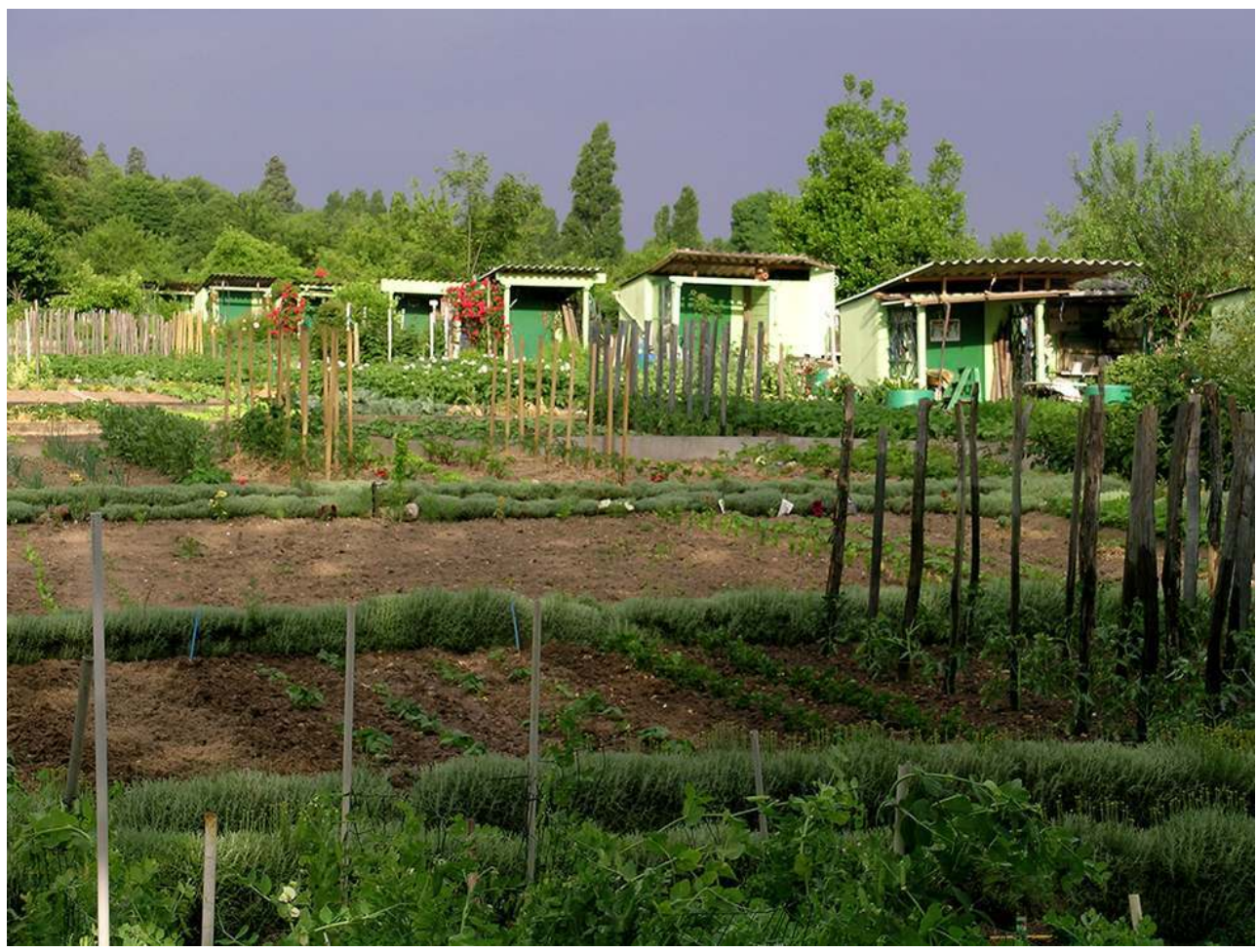

Vue des jardins familiaux de la Figure, à Tours, dont la société gère 511 parcelles. La ville de Tours possède 1300 jardins répartis sur 18 sites et gérés par 13 sociétés différentes.

Phot. Bruno Marmiroli. (c) Bruno Marmiroli.

Gérard Monédiaire, dans un article du Dalloz ${ }^{14}$, pointe la délicate question du droit dès lors que se pose celle du jardin. Sans détailler l'ensemble des lois qui régissent la création, la gestion et la protection des jardins collectifs ${ }^{15}$, il faut mentionner l'éparpillement des différents textes législatifs les concernant au sein de cinq codes distincts: rural, de l'expropriation, de l'urbanisme, de la construction et de l'habitation, des impôts. Une profusion d'articles qui maintient la dissymétrie de traitement entre les jardins familiaux ${ }^{16}$ et les autres. En 2006, la Fédération nationale des jardins familiaux a pris soin d'ajouter le terme « collectif » à l'appellation originelle, pour s'adapter aux nouvelles attentes et élargir son champ d'action. En maintenant la présence d'une fédération encadrant les pratiques, définissant les modes d'investissement des lieux ou orientant les palettes végétales, nous nous éloignons d'une approche collective et partagée du jardin. L'évolution de l'ancienne Ligue n'a rien d'illogique, elle répond à une forme d'institutionnalisation du jardin.

9 Une récente proposition de loi $^{17}$ a tenté, en vain, de poursuivre l'unification des pratiques nouvelles au sein d'une appellation unique de "jardins collectifs", des initiatives regroupées en trois thèmes : les jardins familiaux hérités des jardins ouvriers, les jardins d'insertion ${ }^{18}$ (Jardins de la solidarité, Jardins de cocagne...) et les jardins partagés. L'objectif de la proposition, outre une simplification de la législation, était surtout de permettre aux initiatives nouvelles (la proposition mentionne même l'émergence de mouvements d'appropriation de terrains délaissés) de bénéficier des mêmes avantages que ceux accordés aux jardins familiaux. La nuance n'est pas mince et mérite que l'on s'y arrête. Si le fonctionnement des associations de jardins familiaux se fonde sur une 
division en lots assimilable à un renforcement de la propriété individuelle et que la vie collective résiduelle reste soumise à des règlements inhibiteurs, les variantes contemporaines mentionnées dans la proposition de loi font table rase de la segmentation de l'espace pour investir collectivement les lieux. La gestion collective et participative devient possible, de même que l'organisation de manifestations «de nature socioculturelle» en complément des activités de jardinage. Les dimensions intergénérationnelles et multiethniques sont également mentionnées comme des caractéristiques importantes. Malgré des demandes répétées du sénateur Christian Cointat aux ministres de l'Agriculture qui se sont succédé depuis une dizaine d'années, la proposition de loi n'a jamais passé les portes de l'Assemblée.

10 Remarquons que les avancées en matière législative concernant les droits des jardins familiaux ont été portées par des personnages politiques situés à droite. Le jardin collectif incarnerait plutôt des valeurs ancrées dans une tradition nationale et non pas un réservoir de biodiversité, un écosystème digne d'être protégé. Aucun élu national provenant d'un parti écologiste n'apparaît. Ce n'est qu'à l'échelle locale qu'ils agissent à travers des actions engagées mais ils restent souvent impuissants à protéger les jardins face à la pression foncière, la création d'une zone d'activité ou d'une infrastructure. En l'absence de protection juridique, le jardin, simple réserve foncière, est une quantité négligeable de la fabrication de la ville.

11 L'histoire des jardins ouvriers connaît un tout autre destin outre-Rhin. Les racines ouvrières sont communes, l'abbé Lemire ayant un homologue allemand, le docteur Moritz Schreber (1808-1861), à l'origine des Schrebergärten ${ }^{19}$, dont l'émergence et le développement autour de la ville de Leipzig sont antérieurs à la création de la Ligue. L'essor du mouvement allemand semble puiser dans un tout autre registre que le paternalisme. Il n'est pas question du rôle de l'Église ou de valeurs morales, les différentes associations relevant plus d'initiatives individuelles, parfois politiques, et la solidarité reste un caractère permanent d'un projet qui varie selon les régions, allant jusqu'aux prémices des mouvements écologistes chez les Lebensreformer végétariens de Berlin au tout début du $\mathrm{xx}^{\mathrm{e}}$ siècle. À la fin du $\mathrm{xx}^{\mathrm{e}}$ siècle, les jardins familiaux étaient dix fois plus nombreux en Allemagne qu'en France (1 300000 contre 120 000). Si le cadre règlementaire existe également, il intègre une plus grande liberté concernant la dimension des abris, devenant parfois une annexe de l'habitation principale. Les nombreuses pressions foncières liées à l'extension des métropoles ont conduit à une politisation d'un mouvement pourtant associé, à partir de la seconde moitié du $\mathrm{xx}^{\mathrm{e}}$ siècle, à une mise à l'écart de la vie politique à travers la pratique du jardinage. Les associations ont su se regrouper au sein d'une union fédérale permettant de défendre plus efficacement leurs droits ${ }^{20}$. À titre d'exemple, citons l'association de Potsdam qui, avec l'aide d'un avocat, a gagné son procès contre la Ville pour sauver des parcelles de jardins en 1994. Certains partis politiques ne s'y trompent pas, comme le Parti social-démocrate (SPD), qui a proposé un programme intégrant la question des jardins aux élections de 2002. L'enjeu est d'envergure, près de $5 \%$ de la population allemande ayant accès à un " petit jardin $»^{21}$.

Plus récemment, la question de l'intégration des jardins communautaires au sein de la politique mise en place par la Ville de Berlin s'est posée : si la planification urbaine prend en compte de manière accrue les questions environnementales, allant jusqu'à imposer lors de nouvelles constructions des mesures compensatoires de création d'espaces verts, comment tenir compte des nombreux lopins dont l'usage a progressivement glissé de la 
production potagère au jardin récréatif ? Leurs clôtures, leurs modes de gestion et leurs jardiniers sont-ils compatibles avec la création de nouveaux espaces verts selon les trois thèmes de la Green Vision Berlin: Beautiful city, Productive landscape et Urban nature? Probablement en plaçant le jardin communautaire allemand au sein d'un processus de fabrication collective du cadre de vie.

\section{Urbanisme versus écologie}

Retour en France. Historiquement, les parcelles affectées à la pratique du jardinage collectif sont reléguées à la périphérie, dans des zones non aedificandi pour cause d'inondabilité, de proximité de réseaux ou d'infrastructures... Elles sont parfois associées à des complexes industriels, disséminées aux abords. Il est d'ailleurs intéressant de constater que les jardins reconnus et protégés au titre des monuments historiques sont, dans la plupart des cas, inscrits dans un ensemble plus vaste intégrant l'architecture (cas du Familistère de Guise par exemple) et non pour leurs qualités intrinsèques. L'évolution de l'urbanisme, l'émergence des éco-quartiers, la prise en compte des friches agricoles périurbaines et le développement d'une sensibilité écologique tendraient à leur redonner une certaine légitimité. Les jardins collectifs bénéficieraient d'une visibilité leur permettant de changer momentanément de statut, passant d'une « vocation sociale à une vocation urbanistique $~_{22}$. "Après avoir été un accompagnement des grands ensembles, ils sont aujourd'hui une composante des espaces verts » bien que la dimension sociale n'ait pas disparu totalement. Pour faire l'objet d'un classement en tant que «terrains cultivés à protéger ", les parcelles doivent se trouver en zone urbaine du plan local d'urbanisme ${ }^{23}$. Le Code de l'urbanisme ${ }^{24}$ précise que les parcelles bénéficient d'une protection les rendant inconstructibles. Mais cette classification n'est valable que pour les jardins « familiaux » et les terres agricoles ou les maraîchers (fig. 2). 


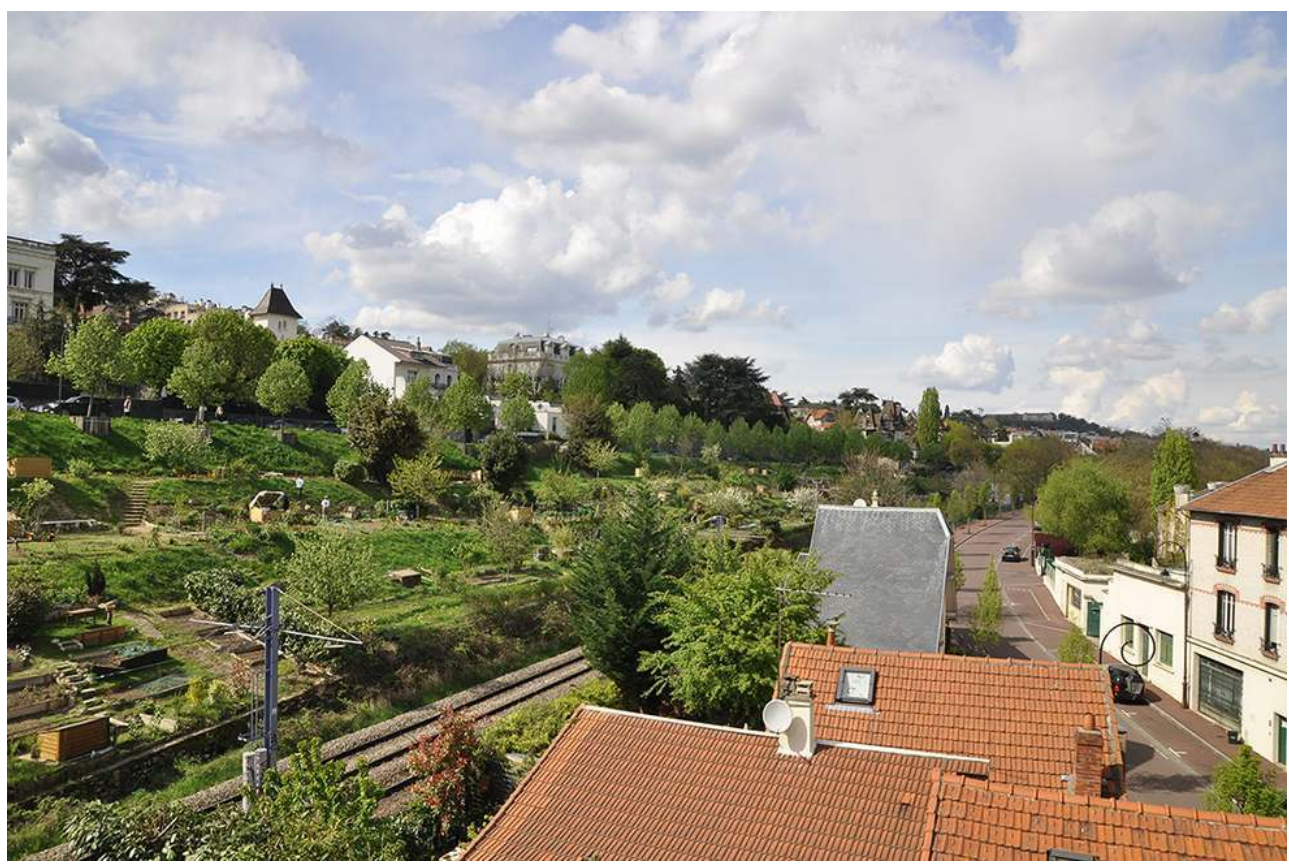

Vue, depuis la passerelle de l'Avre, des jardins familiaux de Saint-Cloud (Hauts-de-Seine) situés entre la voie de chemin de fer et les lotissements du XIX ${ }^{e}$ siècle. Les jardins occupent des parcelles en pente et font office de zone tampon entre la ville et le réseau d'infrastructure.

PHOT. MOONIK, cC. () MOONIK, cc.

Dans tous les cas, l'accès au foncier et la sécurisation des parcelles restent les clefs d'une politique durable de protection et d'expansion des jardins collectifs. Quelle que soit leur nature, leur relégation posent des problèmes aux populations souffrant déjà d'un manque de mobilité, rendant partiellement caduque l'hypothèse d'implantation des jardins sous la forme de «ceintures vertes» aux abords des agglomérations. La notion de continuité entre jardin et habitat constitue un facteur important du succès de l'opération. En complément de la question de l'implantation, à l'échelle d'un territoire d'agglomération, celle de la sécurisation du foncier renvoie à la précarité de bon nombre d'initiatives avec, dans certains cas, la menace permanente de l'expulsion. Si Jérôme Clément, directeur de la Fédération nationale des jardins familiaux et collectifs, qui bénéficie pourtant du cortège législatif le plus protecteur, dénonce le manque de courage politique dans l'application du droit de préemption ${ }^{25}$, que peuvent dire les membres de collectifs non affiliés, ceux qui n'investissent les friches que pour faire vivre un projet sans autre dessein que celui d'œuvrer collectivement, à l'écart de toute tentative de récupération ? Rien non plus, ou si peu, du côté des documents d'urbanisme qui permettraient d'afficher une volonté politique en atténuant la pression foncière autour de terrains convoités qui seraient affectés à des espaces naturels ou des jardins. La Ville de Lyon a même pris le soin de classer les jardins à protéger en espace boisé classé au plan local d'urbanisme, un zonage pourtant réservé aux bois, forêts, parcs, arbres isolés, haies et plantations d'alignement, c'est dire si la législation mériterait d'être adaptée. Certains plans locaux d'urbanisme classent les jardins familiaux dans une sous-rubrique de zone $\mathrm{N}^{26}, \mathrm{Nj}$, impliquant de fait l'existence d'un environnement naturel important aux abords et une absence de pression foncière. Les risques d'effacement sont limités dès lors que la 
collectivité est propriétaire des terrains, mais elle fait rarement le choix de la marge et s'en remet plus aisément aux associations reconnues localement pour gérer les lieux.

En l'absence d'enjeux liés à l'extension urbaine (cas des zones inondables par exemple), il reste la pression liée aux espaces de loisir, terrains de sport, promenades de santé, pistes cyclables... qui entraîne régulièrement un abandon des parcelles auquel succède un constat de friche et le nécessaire retour dans le giron d'une planification urbaine qui n'a jamais pu intégrer les pratiques marginales. Le transfert du vide au vague puis du vague au vert ${ }^{27}$ permet à la collectivité de reprendre la main sur un tissu décousu, incompatible avec un grand projet de ville et foyer ponctuel de mouvements contestataires ou simplement dissidents. Les mots du jardin collectif peinent à infiltrer le discours politique. Rien de tel avec les terrains de sport ou les golfs, ils se parent d'une élégante teinte verte bien éloignée de pratiques respectueuses de l'environnement. Le jardin potager de la Gloriette, à Tours, situé en zone inondable, géré par la collectivité et ouvert à tous, est maintenant cerné par l'extension d'un golf perçu, au moins dans les gazettes communales, comme un espace vert de même nature, implanté au sein d'un complexe plus vaste qualifié d'espace naturel (fig. 3).

Figure 3

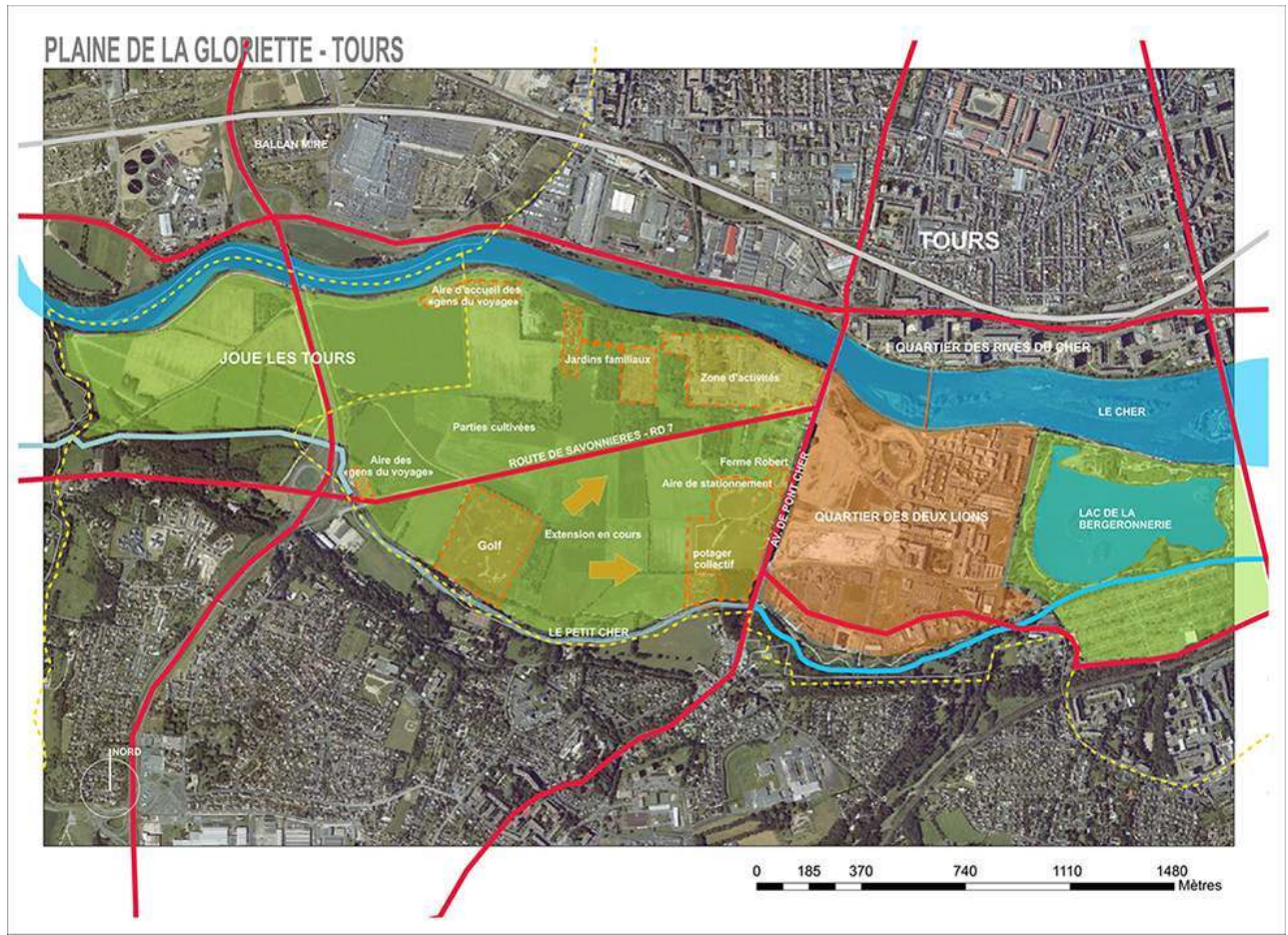

Schéma d'organisation de la plaine de la Gloriette (Tours), un « espace naturel » au cœur de l'agglomération. Peu à peu, les différents équipements sportifs gagnent du terrain sur les parties jardinées collectivement.

Phot. L'Atelier. ( ) L'Atelier.

Les autres actions, plus participatives, plus collectives, doivent intégrer la dimension éphémère au sein du projet de jardin, à moins qu'il ne se sédimente en étant intégré aux politiques publiques. Le jardin de l'îlot d'Amaranthes, à Lyon, a initié une nouvelle approche en proposant le passage du jardin de jardinier au jardin d'art. L'artiste (et jardinier) Emmanuel Louisgrand a réalisé en 2003 une œuvre dont le principe intègre la croissance des végétaux, prenant la forme d'un jardin organisé autour d'une serre ${ }^{28}$ 
(fig. 4). Le visiteur était alors convié à contempler la transformation du lieu, puis à en devenir acteur en jardinant. Éphémère puisque située sur une ancienne friche convoitée, la parcelle est maintenant gérée par une association née en 2004, Brin d'Guill, inspirée des mouvements communautaires de New York.

Figure 4

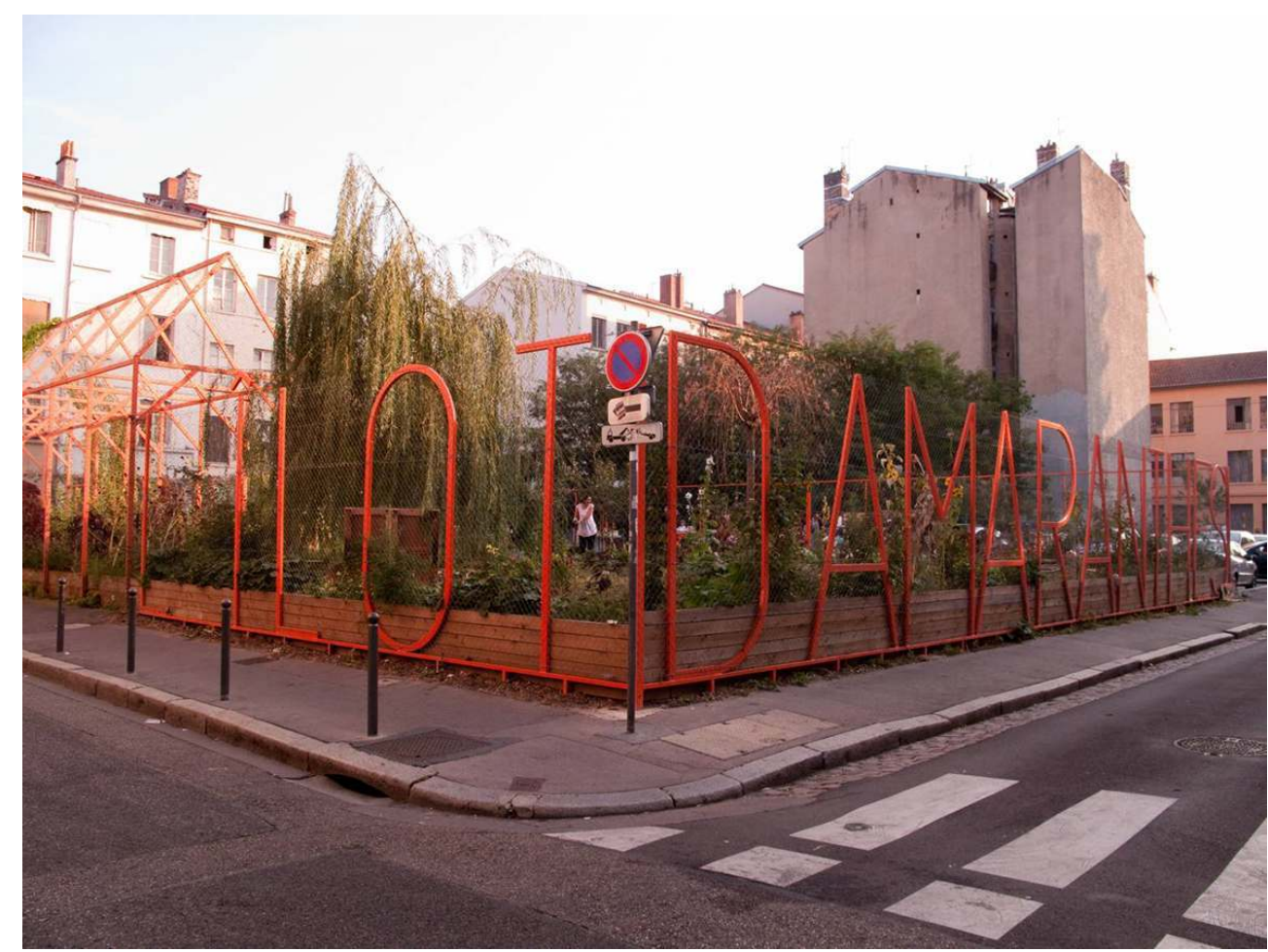

Jardin de l'îlot d'amaranthes, créé à Lyon par Emmanuel Louisgrand et géré depuis 2004 par l'association Brin d'Guill.

Phot. Nicolas Daum. CC. ( ) Nicolas Daum, CC.

17 Les pratiques à la marge, les excès de liberté de choix dans la palette végétale, l'arrivée d'une population étrangère, le choix de l'expression d'un collectif au sein d'une parcelle unique, les expériences plastiques... sont tenus à l'écart des jardins familiaux et ne peuvent s'incarner au sein d'une définition trop exiguë du jardin. Dans le cas de l'expérience de la Fournillère, dans l'agglomération nantaise, Élisabeth Pasquier explique l'esprit d'investissement originel du lieu :

En l'absence de toute règle écrite et $d u$ fait des modalités d'appropriation du terrain, la Fournillère permet de jouer le rôle d'un territoire, support de communauté, non seulement pour les « anciens » mais pour les nouveaux migrants [...] les parcelles ne sont pas closes, voisinages et sociabilités circulent. Les représentations et les pratiques sont plurielles, elles concernent l'ensemble du territoire et tout se passe comme si le territoire de chaque communauté pouvait glisser sur un espace commun ${ }^{29}$.

18 Bien souvent, les jardiniers à l'initiative des squats qui s'implantent dans des interstices urbains, dents creuses, délaissés communaux ou privés, n'ont pas de visibilité sociale. Inconnus des services techniques, ils sont en marge des circuits officiels des jardins familiaux, dont les gestionnaires sont régulièrement taxés de pratiques discriminantes, tant sur le plan social que végétal : «Les jardins classiques sont des lieux où les pratiques 
sont très normées ; ce sont des lieux de domination sociale et culturelle qui redoublent les logiques d'exclusion. ${ }^{30}$ ”

19 À l'échec de l'intégration des individus répond celui de la mixité végétale, seule garante d'une approche réellement environnementale du jardin collectif. Si les mauvaises herbes sont bannies, parfois éradiquées à l'aide de pesticides, aucun jardinier officiel ne saurait donner une définition claire de ces « rudérales ${ }^{31} »$ ou de ces messicoles ${ }^{32}$ indésirables. Ils ne savent ni les nommer, ni en décliner les bienfaits, alors même que ce cortège de plantes possède comme première vertu de nous renseigner sur la nature du sol et comme seconde d'être, le plus souvent, comestible (plantain, ortie dioïque, pissenlit dent-de-lion, laiteron maraîcher, carotte sauvage, mauve, berce commune, lierre terrestre et tant d'autres). Le projet d'inventaire de la flore des villes, porté par le Muséum national d'histoire naturelle et l'association Tela Botanica, permet aux habitants de prendre part au travail de recherche en alimentant une base de données ${ }^{33}$. Ce pas intéressant, qui puise dans l'approche participative propre aux botanistes amateurs, peut éclairer une nouvelle approche partagée de la connaissance de son environnement et, par extension, de son patrimoine en intégrant l'idée d'un contrat naturel lié à la re-connaissance mutuelle des sujets. Pourquoi ne pas envisager de conférer aux plantes un statut (et donc une protection juridique) similaire à celui des animaux ? Faune et flore réunis enfin pour que l'écosystème «jardin» ne soit plus en équilibre précaire mais en évolution postclimacique ${ }^{34}$.

Une reconnaissance écologique du jardin collectif reste à inventer, qui lui procurerait une protection durable en intégrant ses nombreuses aptitudes au sein de l'écosystème "ville». Le prix de cette aventure serait l'abandon de prétentions esthétiques, d'une certaine idée de l'ordre et de l'hygiène, la mise à l'écart de bon nombre de normes et de règlements pour une recherche plus poussée de l'ensemble du cortège faune-flore qui accompagne notre quotidien. La notion de commensalisme ou même de plante compagne, défendue par l'ethnobotaniste Pierre Lieutaghi, pourrait figurer en bonne place dans la liste des préoccupations des communes souhaitant intégrer l'environnement au sein de leurs projets urbains. Hors norme, implantée dans un espace encore non défini par le plan local d'urbanisme en cours, ceinte de constructions et perçue de longue date comme une friche, la parcelle sur laquelle jette parfois son dévolu le jardinier autonomiste constitue un entre-deux urbain dans l'espace et dans le temps. Dans l'espace, puisque le sol, non affecté, non affublé d'un sigle qui marque de façon durable son devenir. Dans le temps, puisque suspendu à des décisions non prises, des incertitudes politiques ou des fluctuations des prix du foncier. Dans cet interstice peut se glisser l'alternative, le « squat jardinier» évoqué par Élisabeth Pasquier lorsqu'elle détaille son expérience à la Fournillère ${ }^{35}$, dont les occupants ont bénéficié pendant vingt ans d'une absence de décision municipale faisant suite à une expropriation et qui s'est achevée, finalement, par une transformation en jardins familiaux municipaux. La conquête des friches devient un enjeu urbain qui s'affranchit des règles classiques qui régissent l'organisation de la ville : le squat jardinier.

21 Finalement, conclut Françoise Dubost dans la préface de l'ouvrage, que la terre soit distribuée par la municipalité ou par une association, l'esprit du squat disparaît. Acquérir un statut, c'est accepter un mode d'altérité recevable parce que suffisamment plié à nos règles.

22 À Schiltigheim (communauté urbaine de Strasbourg) l'association "Droit au jardin » réunissant quelques jardiniers et demandeurs de jardins familiaux a fait échec à la 
menace de destruction des 31 jardins du Muhlwoerth au profit d'une extension des trois terrains de foot déjà existants portée par la commune. Moins chanceux, les jardins collectifs des prairies Saint-Martin, à Rennes, laisseront la place à un parc naturel urbain dont les journaux vantent les vertus écologiques. Cette fois, c'est la pollution des sols et la trop grande quantité de plomb dans les légumes ${ }^{36}$ qui justifient le départ des jardins et des jardiniers qui militaient pour le droit à l'autoproduction. La communication politique, en éradiquant jardins et jardiniers pour en faire un lieu plus accessible aux habitants, n'est plus à un paradoxe près (fig. 5).

Figure 5

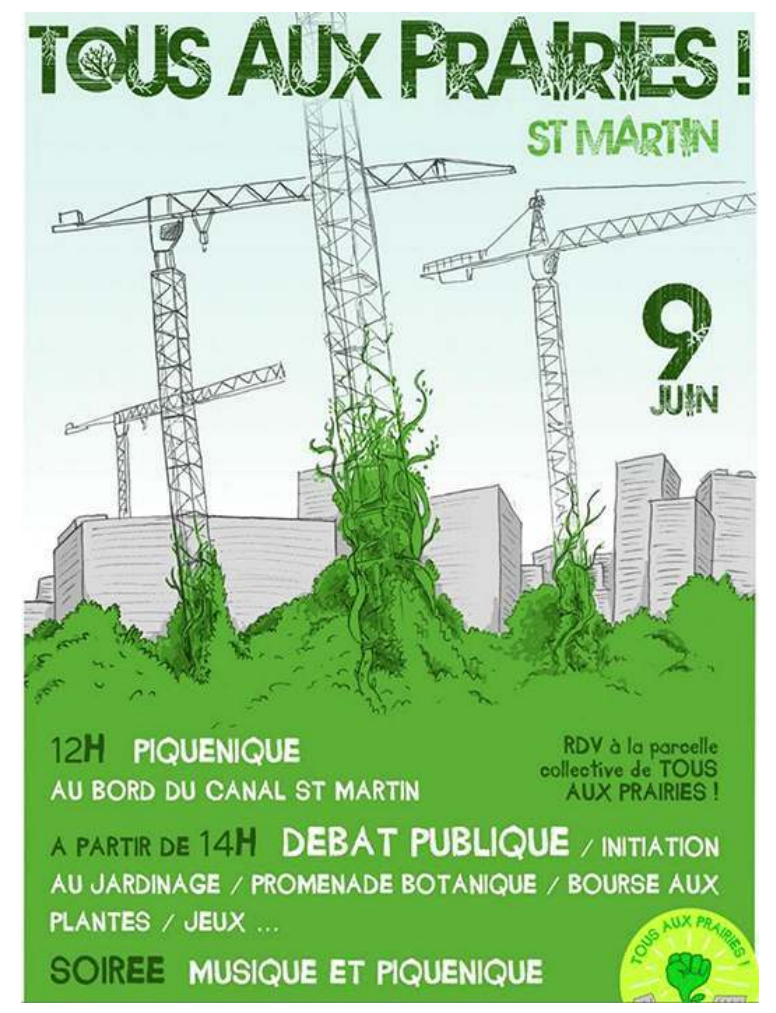

Affiche réalisée par le collectif «Tous aux prairies » créé afin d'empêcher la Ville de Rennes de remplacer les jardins par un parc. DR.

Les jardins familiaux des Tartres (Seine-Saint-Denis), gérés par la société intercommunale des jardins familiaux de Pierrefitte, sont menacés par la réalisation d'une zone d'aménagement concerté de logements. La proposition de déplacement n'est pas souhaitée par les jardiniers. Le projet de zone d'aménagement, intégré au Grand Paris et porté par l'établissement public territorial (ex-communauté d'agglomération) de Plaine Commune, permet de communiquer sur les orientations en matière d'écologie urbaine et décline une série d'actions visant à améliorer la qualité de vie à travers un inventaire de mesures générales allant de l'isolation à la qualité des aliments en passant par la réussite scolaire et l'usage d'espaces publics de qualité favorisant le lien social et la convivialité : «Depuis sa création, Plaine Commune mène une politique volontariste d'écologie urbaine en faveur de la qualité de vie de ses habitants et de ses usagers ${ }^{37}$. " Nouveau paradoxe de la rhétorique lorsque l'acte fondateur de la zone d'aménagement est l'éradication du seul lien tangible existant entre des habitants et leur environnement. 

havre de paix permettant de circonscrire la tentation de la révolte. Le glissement sémantique de familial à collectif n'est pas uniquement destiné à coller à l'évolution sociétale. Le jardin qui émerge, s'installe et permet la production collective dans les interstices urbains incarne et alimente parfois une colère sourde et légitime, renvoyant élus et aménageurs à leurs discours incantatoires. La radicalité des réponses nous écarte de l'idée d'une relation, parfois belliqueuse, parfois harmonieuse, entre le jardinier et son jardin, microcosme à l'équilibre lorsqu'il est bien traité, qui constitue l'essence même de l'écologie, s'affranchit des dogmes de la planification et des tentatives de récupération. La difficulté d'appréhender et d'intégrer la polysémie du jardin dans les politiques publiques en matière d'urbanisme nous renseigne également sur l'incapacité à rendre compte d'une relation équitable et non exclusivement utilitaire entre les individus et la nature ordinaire $^{40}$ (fig. 6). 


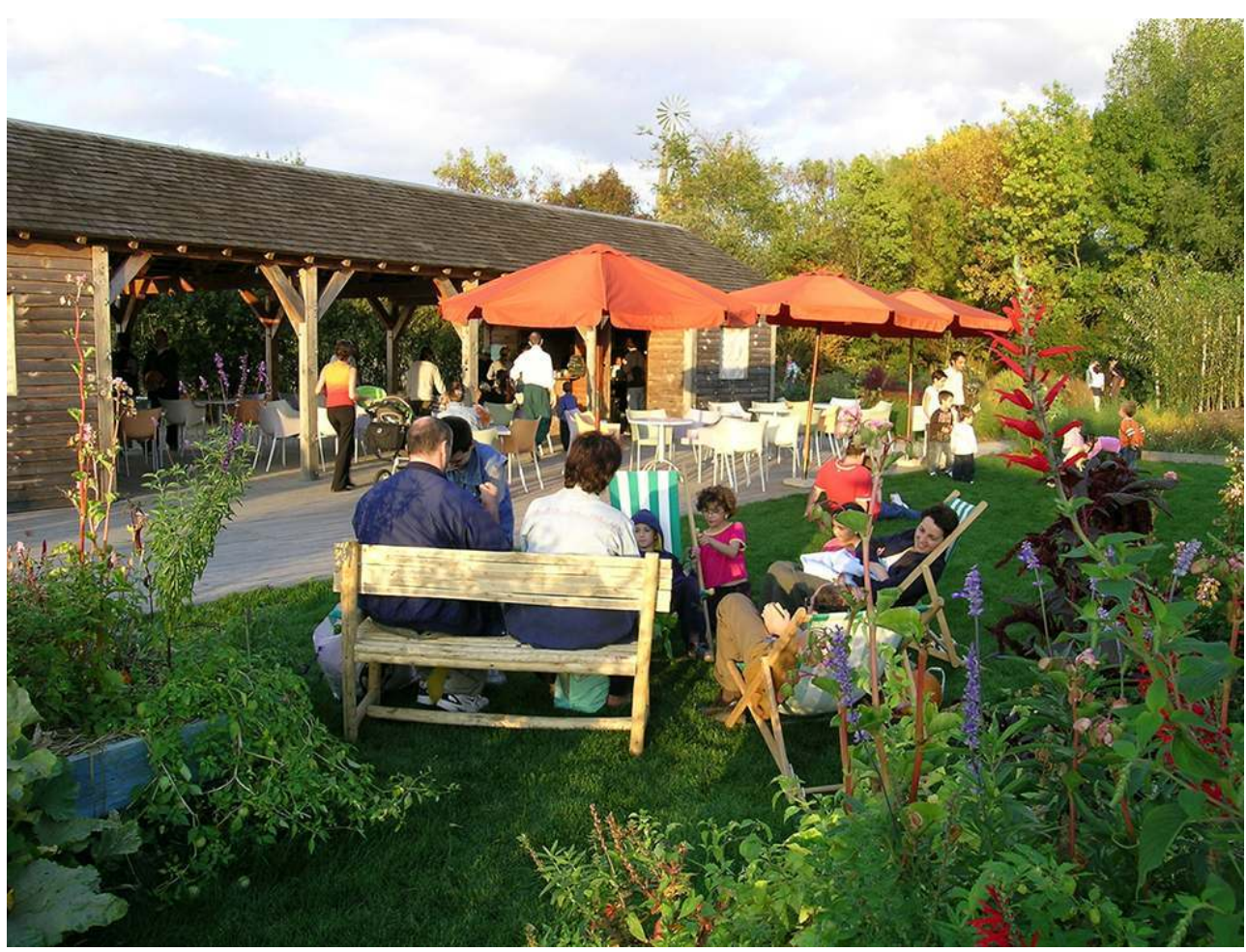

Jardin potager de la Gloriette, à Tours. Créé en 2000, ce jardin potager, ouvert à tous, sert à l'accueil de nombreux ateliers et manifestations autour du végétal.

Phot. Bruno Marmiroli. (c) Bruno Marmiroli.

\section{La question du patrimoine}

En dernier lieu, la dimension patrimoniale des jardins collectifs mérite d'être évoquée à plusieurs titres. Ils agissent comme des cadres de référence pour la préservation et la transmission d'une mémoire collective qui s'inscrit pleinement dans une définition du patrimoine. Selon Sébastien Marot $^{41}$, le jardin se présente comme un médium de sémantisation du paysage. Quelle syntaxe propose le jardin collectif? Le jardin est « un endroit majeur où l'art de la mémoire, progressivement battu en brèche et menacé de marginalisation dans l'économie générale du savoir, a cherché à se renouveler et à se refonder $\aleph^{42}$. La transformation du territoire en paysage " peut être rapportée au projet de constituer des systèmes de lieux et de les rendre disponibles pour la mémoire individuelle et collective ». Les jardins qui procèdent d'une envie collective d'être au monde en offrant un statut aux plantes compagnes et aux jardiniers amateurs, sans préoccupation esthétique mais avec l'idée d'un partage équitable des ressources, constituent un modèle digne de figurer dans la mémoire collective contemporaine du jardin.

Sur un plan moins philosophique, le jardin "partagé » s'inscrit dans une dynamique patrimoniale : un lieu de préservation des connaissances et de diffusion large des savoirs et des pratiques. Un lieu où le patrimoine vit puisque vivant. L'étude ethnographique réalisée en 2014 par Fabienne Boursier sur les jardins familiaux des Petits-Bois ${ }^{43}$, créés en 1906 à Versailles, nous offre une approche du jardin comme «objet patrimonial». 
Premiers jardins familiaux à recevoir le label «Jardin remarquable » en raison de leurs atouts d'ordre historique, environnemental, urbanistique, social et humain, ils ne bénéficient pourtant d'aucune protection juridique spécifique.

Le cas des «petits jardins » de la commune de Beaulieu-les-Loches, en Indre-et-Loire, est intéressant à citer. Ces jardins partagés sont gérés par une association dont les principaux objets sont :

- la sauvegarde de la biodiversité des plantes cultivées à travers leur connaissance, leur culture et leur échange ;

- la valorisation d'un lieu de vie, le rôle dans les loisirs et la vie familiale ;

- l'initiation à la nature et à la protection de l'environnement ;

- la valorisation de la vie sociale, associative et culturelle - un support de solidarité ;

- la gestion de l'espace et la mise en valeur du paysage.

Ces orientations, ambitieuses, sont finalement assez classiques dans le cas de jardins partagés qui se fondent sur un équilibre entre jardin et lien social. Ils sont implantés dans un site qui possède une empreinte historique omniprésente puisqu'ils longent un ancien rempart de la ville, dont le centre ancien est couvert par une aire de mise en valeur de l'architecture et du patrimoine (AVAP) approuvée en 2014. Le site qui accueille les jardins partagés n'est pas directement concerné mais il figure bien dans le secteur S2, qui correspond aux abords paysagers du centre historique de l'AVAP. Le règlement mentionne la qualité et la fragilité du paysage, un « écrin » de jardins souvent bordés de murs en pierre. Il mentionne également la présence de jardins remarquables qui figurent avec une trame spécifique sur le plan de l'AVAP mais rien concernant les jardins partagés, pourtant repérés par la toponymie : jardin des Viantaises. Rien d'assez notable ou de remarquable pour que l'architecte du patrimoine en charge de l'étude estime nécessaire de mentionner la singularité du projet paysager, son ancrage intelligent, sa potentialité au sein d'un parcours de mise en valeur du patrimoine, ce que ne mentionnent d'ailleurs pas non plus les statuts de l'association. Les deux mondes s'ignorent et le développement parallèle de l'un et de l'autre n'est dû qu'à l'intérêt des élus et des réseaux associatifs locaux.

Les jardins collectifs sont au centre d'une grille patrimoniale pourtant assez riche et complexe : leur matrice porte une partie de l'histoire urbaine et sociale du territoire, une vertu qui renseignerait utilement les candidats aux projets urbains. Leur agencement interne rend compte de pratiques sociales et de savoir-faire. Sans omettre l'essentiel, ils permettent à un cortège de plantes et d'animaux de subsister, d'interagir et d'enrichir l'écosystème local. Mais l'approche environnementale ou sociale seule ne suffit pas. Françoise Dubost ${ }^{44}$ mentionne également les notions liées à la transmission d'un héritage, à la préservation des identités locales ou régionales pour que le jardin devienne un « objet patrimonial » en tant que tel. Malgré tout, l'approche reste classique : il faut envisager un rapprochement entre les méthodes qui ont permis de déposer l'architecture vernaculaire sur l'autel du patrimoine national et celles qui pourraient éclairer d'un jour nouveau des jardins ordinaires. L'idée d'un «nouvel objet patrimonial» ne signifie pas une nouvelle méthode d'inventaire. La prise en compte des dimensions évolutives du lieu reste délicate, éloignant pratiquement la notion d'objet en lui substituant l'idée d'un « sujet ». Pour innover, il faut convoquer plusieurs approches. Celle issue de l'étude du patrimoine naturel qui admet qu'il ne peut exister de césure entre une nature exceptionnelle, intacte, préservée dans des «réserves» et une nature ordinaire, brassage permanent, digne d'intérêt, aux multiples atouts et pourtant livré à l'appétit des aménageurs. Une autre, 
qui puise dans l'émergence des études du patrimoine vernaculaire pour définir le jardin, pour reprendre les termes de Françoise Dubost, comme un «lieu de rencontre de nouvelles formes de sensibilité à la nature et de sensibilité au passé ». Et enfin, celle qui relève de l'utopie et qui permettrait de considérer la nature ordinaire pour ses qualités extraordinaires, imposant aux spécialistes de l'« objet » patrimonial un nouveau « sujet » d'étude délicat à saisir. En croisant les approches, en mélangeant les influences et en décloisonnant les pratiques pour nous permettre de bénéficier de l'apport des sciences sociales dans la construction d'une approche patrimoniale innovante, nous serions alors en mesure de rejoindre le jardin collectif dans sa capacité d'adaptation pour lui octroyer, enfin, un statut.

\section{NOTES}

1. - MARMIROLI, Bruno. « De quel droit jardine-t-on ». Dans TERRIN, Jean-Jacques (dir.). Jardins en ville, villes en jardin. Marseille: Parenthèses, 2013, p. 282.

2. - VADROT, Claude-Marie. La France au jardin. Histoire et renouveau des jardins potagers. Paris : Delachaux et Niestlé, 2009, p. 58.

3. - Rapport du quatrième congrès des jardins ouvriers, compte rendu rédigé par l'abbé Lemire, 1912.

4. - Ibid., p. 41.

5. - Loi du 12 avril 1906.

6. - Terme utilisé pour qualifier le « chef de famille » auquel est confiée l'exploitation du lopin de terre.

7. - Loi du 26 juillet 1952.

8. - La loi du 31 octobre 1941 clarifie le statut des jardins ouvriers, en distinguant également les jardins industriels et familiaux.

9. - Exemple du règlement 2013 des jardins familiaux d'Annemasse, article 20. Extrait du règlement des jardins familiaux de Saint-Dizier, affiliés à la Ligue et datant de 2008 :

Article 8 - Cultures

$1^{\circ}$ Entretien de la parcelle

Pendant la période de végétation les jardins doivent être tenus propres et en bon état. La plantation de légumes se fera à $20 \mathrm{~cm}$ à l'intérieur des limites du jardin. La totalité du terrain doit être cultivée. Le bénéficiaire d'un jardin non entretenu et envahi de mauvaises herbes à la date du 30 mars recevra une lettre d'avertissement (sauf cas de force majeure justifiée). Si ce premier avertissement n'est pas suivi d'effet dans les quinze jours, un avis de retrait du jardin dans les 24 heures lui sera notifié par lettre recommandée avec accusé de réception.

10. - Jean Royer a été maire de Tours de 1959 à 1995, député d'Indre-et-Loire de 1958 à 1993 et deux fois ministre. À l'origine de grands projets urbains (quartier des Rives du Cher et des Fontaines), son implication, paradoxale, dans la protection des jardins familiaux correspond à une période de remise en question des projets urbains tendant à effacer les traces des installations humaines.

11. - SAFER : société d'aménagement foncier et d'établissement rural, détentrice du droit de préemption des terrains agricoles. 
12. - TARDIF, Pierre. «D'hier à demain... Les jardins ouvriers et familiaux et les pouvoirs publics : de l'isolement au partenariat ». Dans CABEDOCE, Béatrice et PIERSON, Philippe (dir.). Cent ans d'histoire des jardins ouvriers, 1896-1996. La Ligue française du Coin de terre et du Foyer. Grane: Créaphis, 1996.

13. - Ibid., p. 203.

14. - MONÉDIAIRE, Gérard. «Le droit français des jardins familiaux ». AJDI (Actualité juridique du droit immobilier), mars 1994, p. 192-206.

15. - La définition du jardin collectif prend en compte les espaces jardinés hors des limites privées des habitations individuelles ou des espaces verts gérés par une commune et intègre à la fois les jardins ouvriers, familiaux, d'insertion, associatifs, pédagogiques ou partagés gérés par un collectif, organisé (personne morale) ou non.

16. - La définition des jardins familiaux est encadrée par deux articles : art. L. 561-1 et L. 561-2 du Code rural: «Peuvent être dénommés "jardins familiaux» des terrains divisés en parcelles, lesquelles sont affectées à des particuliers y pratiquant le jardinage pour leurs propres besoins et ceux de leur famille, à l'exclusion de tout usage commercial. Tous les jardins répondant à ces critères, quelle que soit leur dénomination, sont assimilés à des jardins familiaux. ». L'affectation d'une parcelle résulte du contrat d'adhésion à l'association qui est chargée de gérer le groupe de jardins familiaux considéré et éventuellement d'y entreprendre des actions pédagogiques et de vulgarisation horticole.

17. - Proposition de loi de 2002 relative aux jardins familiaux et aux jardins d'insertion présentée au Sénat par le sénateur Christian Cointat. Rapport du Sénat $n^{\circ} 376$.

18. - Les jardins d'insertion ont été reconnus et réglementés par la loi d'orientation du 29 juillet 1998 relative à la lutte contre les exclusions, ainsi que par ses textes d'application, qui les intègrent dans le cadre plus large des « chantiers d'insertion ».

19. - Le nom a en fait été donné de façon posthume par Ernst Hauschild, fondateur de l'association de gestion des jardins en 1864, reprenant une partie des travaux du docteur Schreber.

20. - PAILHES, Anne-Marie. «Du jardin ouvrier au jardin familial est-allemand: de la culture ouvrière à la culture précaire ». Dans HERBET, Dominique (éd.). Culture ouvrière/Arbeiterkultur, mutations d'une réalité complexe en Allemagne du XIX ${ }^{e}$ au XXI ${ }^{e}$ siècle. Villeneuve-d'Ascq: Presses universitaires du Septentrion, 2011, p. 209-222, ici p. 214.

21. - Le terme de Kleingarten (petit jardin) a rapidement remplacé celui de Schrebergarten et effacé son origine ouvrière pour ne garder qu'une référence à la taille de la parcelle et à la modestie de l'entreprise.

22. - GUYON, Frédérick. « Les jardins familiaux aujourd'hui : des espaces socialement modulés ». Espaces et sociétés, 2008/3, n 134, "Repenser l'espace et le politique », p. 131-147. Voir le site : https://www.cairn.info/revue-espaces-et-societes-2008-3-page-131.htm [consulté le 22/11/2018]. 23. - Le plan local d'urbanisme (PLU), qui remplace le plan d'occupation des sols, est le document permettant de planifier l'urbanisme au niveau communal, dans le cadre de l'application de la loi relative à la solidarité et au renouvellement urbains, dite loi SRU, du 13 décembre 2000.

24. - Article L 123-1 du Code de l'urbanisme.

25. - CLÉMENT, Jérôme. "Les problèmes et perspectives du point de vue de la FNJFC». Réseau international, thématique développement durable des villes : le rapport entre l'urbain et la nature. Paris, 22 janvier 2009.

26. - Zones $\mathrm{N}$, zones naturelles et forestières du plan local d'urbanisme. $\mathrm{Nj}$ fait référence à une zone $\mathrm{N}$ possédant des jardins.

27. - Thème évoqué dans BÉGUIN, François. «Vagues, vides, verts ». Le Visiteur, $\mathrm{n}^{\circ} 3$, automne 1997, p. 56-69.

28. - Voir dans ce numéro : PRUNET, Camille. «Le jardin-œuvre, une autre façon d'appréhender le jardin collectif autour du travail de l'artiste Emmanuel Louisgrand», In Situ [En ligne], 
37|2018, mis en ligne le 12 décembre 2018, consulté le 19 décembre 2018. URL: http:// journals.openedition.org/insitu/19446.

29. - PASQUIER, Élisabeth. Cultiver son jardin. Chroniques des jardins de la Fournillère, 1992-2000. Paris : L'Harmattan, 2001, p. 135.

30. - CÉRÉZUELLE, Daniel, directeur de recherche au Programme Autoproduction et Développement social - PADES. «Modes informels de jardinage collectif». Réseau international, thématique développement durable des villes: le rapport entre l'urbain et la nature. Paris, 22 janvier 2009.

31. - En parlant d'un végétal, qui pousse sur les décombres (CNRTL).

32. - Plantes « habitant dans les moissons » (Tela Botanica).

33. - Base de données en ligne du Muséum: http://sauvagesdemarue.mnhn.fr/ [consulté le 10/12/2018].

34. - Si le climax correspond à un état d'équilibre d'un milieu, la notion post-climacique fait référence au développement de végétaux au-delà de ce point d'équilibre, introduisant de nouveaux facteurs.

35. - PASQUIER, Élisabeth. Op. cit.

36. - Information provenant du site internet de la métropole rennaise : www.metropole.rennes.fr [consulté le 22/11/2018].

37. - Information provenant du site internet de Plaine Commune: www.plainecommune.fr [consulté le 22/11/2018].

38. - Loi du 24 mars 2014 pour l'accès au logement et un urbanisme rénové.

39. - Plan local d'urbanisme de Clermont-Ferrand, approuvé le 4 novembre 2016, réalisé par l'agence DBW.

40. - La nature ordinaire est entendue comme un espace de nature domestiquée, un écosystème cultivé.

41. - MAROT, Sébastien. «L'art de la mémoire, le territoire et l'architecture ». Le Visiteur, $\mathrm{n}^{\circ} 4$, juin 1999.

42. - Ibid., p. 124.

43. - BOURSIER, Fabienne. Jardins familiaux et jardiniers des Petits-Bois à Versailles: perception et appropriation du lieu. Rapport final, janvier 2016. Voir dans ce numéro : BOURSIER, Fabienne. «Les jardins familiaux des Petits-Bois à Versailles : un patrimoine vivant à préserver ", In Situ [En ligne], 37 |2018, mis en ligne le 12 décembre 2018, consulté le 19 décembre 2018. URL : http:// journals.openedition.org/insitu/18892. Voir également : DESNOS, Pierre. «Des jardins familiaux centenaires labellisés : quel avenir? L'exemple du site Paul Philippe à Versailles », In Situ [En ligne], 37 |2018, mis en ligne le 12 décembre 2018, consulté le 19 décembre 2018. URL : http:// journals.openedition.org/insitu/18798.

44. - DUBOST, Françoise. Vert patrimoine, la constitution d'un nouveau domaine patrimonial. Paris : Éd. de la Maison des sciences de l'homme, 1994.

\section{RÉSUMÉS}

Les jardins collectifs, qui regroupent différents types de jardins, sont porteurs de nouveaux enjeux en matière d'environnement, de lien social, d'échanges et de protection de la nature « ordinaire » dans les territoires métropolitains. Souvent portés par des aspirations collectives 
spontanées qui échappent aux politiques publiques, ils deviennent des espaces institutionnalisés dès lors qu'ils font l'objet d'une prise en charge par la collectivité. Présents dans les discours politiques, ils sont pourtant bien absents des projets urbains, en particulier dans les espaces soumis à une forte pression foncière. Les jardins collectifs ne font pas l'objet de protections à la hauteur des services qu'ils rendent. En reprenant l'histoire des jardins familiaux, qui bénéficient d'une certaine reconnaissance, l'article propose d'étudier les mesures qui permettraient de mieux intégrer les dispositifs de jardins collectifs dans les projets urbains, selon plusieurs orientations. La première relève du droit et permettrait de conférer aux jardins des protections juridiques spécifiques limitant leur précarité. La seconde est liée à l'essor d'un mouvement lié à l'écologie urbaine, dans lequel le jardin a toute sa place malgré les difficultés à intégrer les outils de planification. La dernière orientation est fondée sur un élargissement du champ patrimonial qui permettrait d'y inclure le jardin collectif comme espace médian entre nature et culture.

Collective gardens, a term which covers many different types of gardens, raise new issues around the environment, social links, exchanges and the protection of 'ordinary' nature in metropolitan territories. These gardens are often initiated by spontaneous collective aspirations which ignore public policies. But they become institutionalised spaces once the local authorities take over responsibility for them. Although they may feature in politicians' declarations, they rarely feature in urban projects, particularly where space is subjected to intense real estate pressure. These collective gardens then do not enjoy the protection they deserve in view of the services they provide. By taking a new look at the history of family gardens, which enjoy some measure of recognition, this article proposes further measures that might be studied in order to better integrate collective gardens into urban projects. Several approaches are considered, the first looking at legal measures that could give these gardens specific statutory protection that would limit their precarity. The second approach looks at the developing movement for urban ecology, in which the garden has an important role to play, despite the difficulties of introducing it to town-planning tools. The last orientation is founded on an extension of our notions of heritage in order to encompass the collective garden as a king of median space between nature and culture.

\section{INDEX}

Keywords : collective garden, community garden, town-planning, law, know-how, plants, environment, pratices, inhabitants

Mots-clés : jardin collectif, urbanisme, droit, savoir-faire, plantes, planification urbaine, environnement, pratiques, habitants

\section{AUTEUR}

\section{BRUNO MARMIROLI}

Architecte - paysagiste, directeur de la Mission Val de Loire patrimoine mondial marmiroli@mission-valdeloire.fr 